Benítez-Jiménez, A.; Falces-Prieto, M.; García-Ramos, A. (2020). Jump Performance after Different Friendly Matches Played on Consecutive Days. Revista Internacional de Medicina y Ciencias de la Actividad Física y el Deporte vol. 20 (77) pp. 185-196 Http://cdeporte.rediris.es/revista/revista77/artrendimiento1119.htm

DOI: $10.15366 /$ rimcafd2020.77.012

\title{
ORIGINAL
}

\section{RENDIMIENTO DEL SALTO TRAS VARIOS PARTIDOS DE FÚTBOL DISPUTADOS EN DÍAS CONSECUTIVOS}

\section{JUMP PERFORMANCE AFTER DIFFERENT FRIENDLY MATCHES PLAYED ON CONSECUTIVE DAYS}

\author{
Benítez-Jiménez, A. ${ }^{1}$; Falces-Prieto, M. ${ }^{1,2}$ y García-Ramos, A. ${ }^{3}$ \\ ${ }^{1}$ Licenciado en Ciencias de la Actividad Física y del Deporte. Departamento de Optimización del \\ Rendimiento Físico, Fundación Marcet, Barcelona (España) abeji 88@hotmail.com \\ 2 Doctorando en Ciencias de la Actividad Física y el Deporte, Universidad Pablo de Olavide, \\ (España). Departamento de Optimización del Rendimiento Físico, Fundación Marcet, Barcelona \\ (España) mfalpri@gmail.com \\ ${ }^{3}$ Doctor en Ciencias del Deporte. Facultad de Ciencias del Deporte. Departamento de Ciencias \\ del Deporte y Acondicionamiento Físico, Facultad de Educación, CIEDE, Universidad Católica \\ de la Santísima Concepción, Concepción (Chile) Departamento de Educación Física y \\ Deporte, Facultad de Ciencias del Deporte. Universidad de Granada, Granada (España) \\ amagr@ugr.es
}

Código UNESCO: 5899 Educación Física y Deportes / Physical Education and Sport

Clasificación Consejo de Europa: 17. Otras: Rendimiento deportivo / Others: Sports performance

Recibido 8 de abril de 2018 Received April 8, 2018

Aceptado 1 de octubre de 2018 Accepted October 1, 2018

\section{RESUMEN}

Los objetivos del estudio fueron determinar el cambio en la altura alcanzada en el salto con contramovimiento (CMJ) tras tres partidos amistosos jugados en días consecutivos, y evaluar si existe una asociación significativa entre los cambios en la altura del CMJ respecto a los minutos disputados y los valores de percepción subjetiva del esfuerzo (RPE). La altura de salto de 22 jóvenes futbolistas varones (edad: 16,6 $\pm 0,8$ años) fue determinada en 5 ocasiones: Previaje, Pre-partido 1, Pre-partido 2, Pre-partido 3, y Post-viaje. Los resultados mostraron una reducción de la altura de salto respecto al pre-viaje $(P<0,001$; tamaño de efecto $\geq 1.47$ ), mientras que el cambio en la altura de salto no estuvo 
significativamente correlacionado con los minutos disputados ( $r=-0.02 ; P=0.464)$ ni con la RPE $(r=0.15 ; P=0.249)$. La sucesión de partidos de fútbol provoca una disminución en la altura de salto que no está relacionada con los minutos disputados ni la RPE.

PALABRAS CLAVE: fatiga, CMJ, RPE, fútbol.

\section{ABSTRACT}

The aims of the study were to determine the change in countermovement jump (CMJ) after three friendly matches played on consecutive days, and to evaluate if there is a significant correlation between the changes observed in CMJ height with respect to the minutes played and the rate of perceived exertion (RPE) values. The CMJ height of 22 young male soccer players (age: $16.6 \pm 0.8$ years) was determined in 5 occasions: pre-trip, pre-game 1, pre-game 2, pre-game 3, and post-trip. The results showed a reduction in jump height when compared to the pre-trip $(P<0,001$; effect size $\geq 1.47)$, while the change in jump height was not significantly correlated with the minutes played $r=-0.02 ; P=0.464$ ) nor with the RPE values ( $r=0.15 ; P=0.249)$. The succession of football matches promotes a decrease in jump height that is not related to the minutes played nor to the RPE values.

KEY WORDS: fatigue, CMJ, RPE, soccer.

\section{INTRODUCCIÓN}

El fútbol es un deporte acíclico de naturaleza intermitente en el que el rendimiento está altamente influenciado por las acciones que se realizan a alta intensidad (Faude, Koch, \& Meyer, 2012). Durante los partidos, los jugadores realizan una serie de acciones a alta intensidad entre las que destacan sprints, aceleraciones, desaceleraciones, cambio de dirección, saltos, tackles y golpeos de balón (De Hoyo et al., 2016; Marqués-Jiménez, Calleja-González, Arratibel, Delextrat, \& Terrados, 2017). La sucesión de estas acciones provocan una serie de alteraciones en el sistema músculoesquelético, nervioso, metabólico e inmune (De Hoyo et al., 2016) que conducen a la fatiga de los futbolistas (Brownstein et al., 2017; Thomas, Dent, Howatson, \& Goodall, 2017). Es conocido que la fatiga inducida por la disputa de partidos de fútbol puede disminuir el rendimiento físico durante varios días posteriores a la competición (Ispirlidis et al., 2008; Roe et al., 2017). La alta densidad competitiva actual, donde jugadores de élite pueden llegar a jugar dos o tres partido en una misma semana (Marqués-Jiménez et al., 2017), y la realización de viajes largos en situaciones poco confortables, son situaciones que también pueden afectar al rendimiento de los jugadores (Fowler, Duffield, \& Vaile, 2015). Sin embargo, la fatiga inducida por partidos disputados en días consecutivos en edades jóvenes, donde es frecuente que disputen torneos competitivos, ha sido poco estudiada. 
Los científicos del deporte y preparadores físicos suelen realizar tests para evaluar el estado neuromuscular de los deportistas. El salto con contramovimiento (CMJ) ha sido uno de los tests más utilizados para determinar el rendimiento neuromuscular de los miembros inferiores tanto en deportes individuales como colectivos (Claudino et al., 2017). En fútbol, las altas demandas físicas provocan fatiga neuromuscular durante determinadas fases del partido, así como en la fase final de cada tiempo reglamentario (Silva et al., 2017), produciendo una disminución de la altura del salto inmediatamente después del partido (Marqués-Jiménez et al., 2017), a las 24 horas (Roe et al., 2017), 48 horas (Watkins et al., 2017), 72 horas (Marqués-Jiménez et al., 2017; Roe et al., 2017; Silva et al., 2017) e, incluso, a las 96 horas post-partido (Thomas et al., 2017). Sin embargo, en algunas investigaciones no se han encontrado una correlación entre fatiga neuromuscular inducida por el ejercicio y la disminución de la altura del CMJ (Krustrup, Zebis, Jensen, \& Mohr, 2010; Thorlund, Aagaard, \& Madsen, 2009). Por tanto, podría ser interesante analizar si la altura alcanzada en el CMJ puede ser capaz de detectar la fatiga neuromuscular inducida por la disputa de varios partidos en días consecutivos y por un viaje de larga duración poco confortable.

En deportes de equipo es muy importante realizar un control individual de las cargas de entrenamiento para valorar el estímulo de entrenamiento (CamposVazquez, Toscano-Bendala, Mora-Ferrera, \& Suarez-Arrones, 2017), dado que un estímulo excesivo incrementa el riesgo de sufrir una lesión y uno insuficiente disminuye el rendimiento (Vanrenterghem, Nedergaard, Robinson, \& Drust, 2017). La carga de entrenamiento puede ser dividida en dos sub-secciones: carga externa (estímulo de entrenamiento realizado) y carga interna (estrés fisiológico) (Malone et al., 2015). En fútbol, para la cuantificación de la carga interna se han utilizado varios métodos asociados a la frecuencia cardíaca como el impulso de entrenamiento (Banister, 1991), impulso de entrenamiento individual (iTRIMP) (Manzi, Bovenzi, Franco Impellizzeri, Carminati, \& Castagna, 2013), método de Edwards (Edwards, 1993), entre otros, aunque solamente son utilizados por equipos con grandes recursos económicos (Campos-Vazquez et al., 2017; Gil-Rey, Lezaun, \& Los Arcos, 2015). Una alternativa económica a estos métodos de cuantificación de la carga de entrenamiento es la percepción subjetiva del esfuerzo (RPE). La RPE es fácil de usar e indica el estrés físico y psicológico de los jugadores (Vanrenterghem et al., 2017). Sin embargo, al ser subjetivo, en acciones de alta intensidad con amplios períodos de recuperación este valor de esfuerzo puede ser subestimado o sobreestimado (Scott, Lockie, Knight, Clark, \& Janse de Jonge, 2013). Este hecho hace que sea interesante analizar si la RPE obtenida post-partidos podría modular los cambios esperados en el CMJ a consecuencia de partidos consecutivos en jóvenes futbolistas.

Para aportar luz a lo anteriormente expuesto, este estudio fue diseñado para ver como afectaba la disputa de varios partidos amistosos en días consecutivos a la fatiga neuromuscular evaluada por el decremento en altura de salto. Específicamente, los objetivos del estudio fueron (1) determinar la evolución del cambio en la altura alcanzada en el CMJ tras varios partidos amistosos jugados en días consecutivos, y (2) evaluar si existe una asociación significativa entre los 
cambios observados en la altura del CMJ respecto a los minutos disputados y los valores de RPE. Nuestra primera hipótesis fue que la altura del CMJ disminuiría con la sucesión de partidos. Nuestra segunda hipótesis fue que los cambios en la altura de salto estarían relacionados con la acumulación de minutos totales y los valores de RPE medios post-partidos. Los resultados esperados podrían ser importantes porque proporcionarían a los entrenadores una orientación acerca del deterioro neuromuscular a consecuencia de la disputa de partidos en días consecutivos, ayudando ello a prevenir lesiones por exceso de carga o fatiga.

\section{MÉTODO}

\section{Participantes}

Veintidós jóvenes futbolistas varones (edad: $16,6 \pm 0,8$ años; masa corporal: 68,3 $\pm 6,5 \mathrm{~kg}$; altura: $175,0 \pm 6,6 \mathrm{~cm}$ ) participaron en el estudio de forma voluntaria. Todos los participantes tenían al menos 2 años de experiencia en la realización del salto con contramovimiento (CMJ) y en la utilización de la escala de percepción subjetiva del esfuerzo (RPE). Ninguno de los participantes analizados presentó lesiones previas que pudieran comprometer la realización del test de salto. Los participantes fueron informados del procedimiento a realizar $y$, al ser menores de edad, los padres firmaron un formulario de consentimiento antes de comenzar la investigación. El protocolo de estudio se adhirió a los principios de la Declaración de Helsinki y fue aprobado por el comité de ética institucional.

\section{Diseño experimental}

Este estudio fue diseñado para analizar la evolución de la fatiga inducida por un viaje de larga duración en autobús y tres partidos amistosos jugados en días consecutivos. La altura de salto alcanzada durante el CMJ se utilizó como un indicador de fatiga neuromuscular (Claudino et al., 2017). La altura de salto se determinó en 5 ocasiones: (1) Pre-viaje (en el lugar de residencia antes de realizar un viaje en autobús de 15 horas), (2) Pre-partido 1 (4 horas antes del partido 1), (3) Pre-partido 2 (4 horas antes del partido 2), (4) Pre-partido 3 (4 horas antes del partido 3), y (5) Post-viaje (en el lugar de residencia 48 horas después de haber disputado el partido 3 y haber realizado el viaje de regreso en autobús durante 15 horas) (Figura 1). Todas las evaluaciones fueron realizadas a la misma hora del día y bajo similares condiciones meteorológicas $\left(\sim 22^{\circ} \mathrm{C}\right.$ y $\sim 60 \%$ humedad). 
Figura 1. Representación gráfica del protocolo experimental.

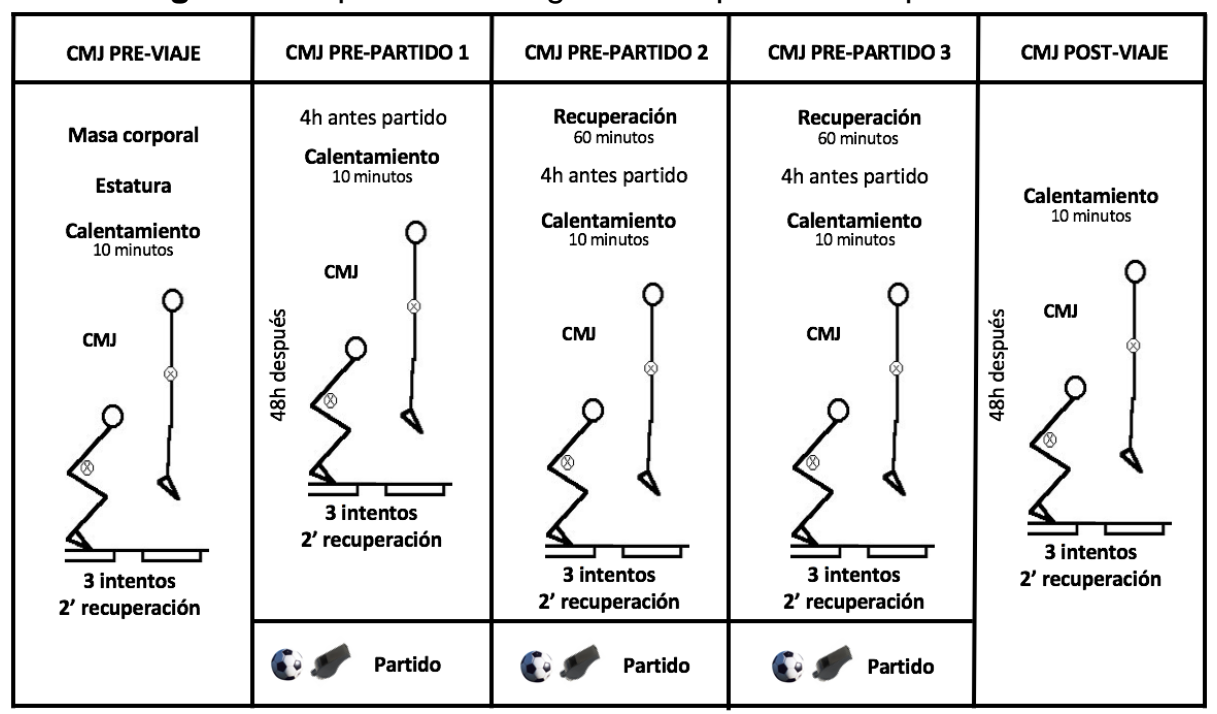

\section{Procedimiento}

Los participantes realizaron un calentamiento estandarizado de 10 minutos antes de realizar los CMJ de evaluación. El calentamiento incluyó carrera continua a baja intensidad, estiramientos dinámicos, ejercicios de movilidad articular, y finalmente $6 \mathrm{CMJ}$ realizados en orden creciente de intensidad. Tras el calentamiento, los participantes realizaron $3 \mathrm{CMJ}$ sobre una plataforma de contacto (Chronojump-Boscosystem, Barcelona, España) y fueron instruidos a saltar lo más alto posible tras alcanzar una angulación de rodilla de $\sim 90 \%$. Los participantes fueron instruidos a mantener las manos en sus caderas durante la ejecución del CMJ y a aterrizar con las piernas extendidas y con máxima flexión plantar. Si algunos de estos requisitos no fueron cumplidos, el intento se repitió. Para minimizar los efectos de la fatiga, 2 minutos de descanso se implementaron entre saltos sucesivos (Jiménez-Reyes et al., 2017).

La primera sesión de salto fue realizada 48 horas antes de dar comienzo el primer partido amistoso en las instalaciones donde habitualmente entrenaban los participantes. La segunda sesión de salto estuvo precedida por un viaje en autobús con una duración de 15 horas, en unas instalaciones diferentes a las de la primera evaluación. La segunda, tercera y cuarta sesión de salto fueron evaluadas 4 horas antes de cada partido amistoso, que fueron jugados en días consecutivos. La última sesión de salto tuvo lugar en las instalaciones donde entrenaban 48 horas después del último partido amistoso y después de otro viaje en autobús de 15 horas.

Las diferentes evaluaciones del CMJ se realizaron sobre una superficie dura de similares características en ambas instalaciones y fueron ejecutadas por los participantes con zapatillas de deporte. 
La escala de percepción subjetiva del esfuerzo de Borg de 0-10 (Borg, Hassmén, \& Lagerström, 1987) fue pasada a los jugadores 30 minutos después de completar el partido para garantizar que el esfuerzo percibido no fuese agudo, sino referente a los minutos disputados (Campos-Vazquez et al., 2017).

\section{Análisis estadístico}

Los datos se presentan como media \pm desviación estándar. Antes de cualquier análisis estadístico, la distribución normal de los datos (test de Shapiro-Wilk) y la homogeneidad de las varianzas (test de Levene) fueron confirmadas $(P>$ 0.05 ). Un análisis de varianza (ANOVA) de medidas repetidas con la corrección post-hoc de Bonferroni fue aplicado para explorar las diferencias en altura de salto entre los diferentes puntos de medida (pre-viaje, partido 1, partido 2, partido 3 , post-viaje). El tamaño del efecto (TE) de Cohen se calculó para cuantificar la magnitud de las diferencias. El criterio para interpretar la magnitud del TE fue el siguiente: trivial $(<0.2)$, pequeño $(0.2-0.6)$, moderado $(0.6-1.2)$, grande $(1.2-$ 2.0) y extremadamente grande (> 2.0) (Hopkins, Marshall, Batterham, \& Hanin, 2009). La asociación entre el cambio en la altura de salto (post-viaje - pre-viaje) con el número total de minutos disputados y la RPE media de los 3 partidos se cuantificó a través del coeficiente de correlación de Pearson ( $r$ ). Además, un análisis de regresión lineal múltiple se empleó para explorar la posibilidad de predecir el cambio en la altura de salto a partir del número total de minutos jugados y la RPE media de los 3 partidos disputados. Todos los test estadísticos fueron realizados a través del software SPSS (versión 22.0: SPSS, Inc., Chicago, IL, USA). La significación estadística se fijó para un $P<0.05$.

\section{RESULTADOS}

El ANOVA reflejó la existencia de diferencias significativas en la altura de salto entre los diferentes puntos de medida $\left(F=55.7, P<0.001, \eta_{p}^{2}=0.726\right)$. Las comparaciones post-hoc de Bonferroni revelaron que la altura de salto pre-viaje $(41.4 \pm 3.9 \mathrm{~cm})$ fue significativamente superior que en partido $1(35.7 \pm 3.7 \mathrm{~cm}$; $P<0.001$; TE $=-1.47)$, partido $2(33.4 \pm 3.8 \mathrm{~cm} ; P<0.001$; TE $=-2.07)$, partido $3(35.5 \pm 2.9 \mathrm{~cm} ; P<0.001 ;$ TE $=-1.53)$ y post-viaje $(35.4 \pm 3.6 \mathrm{~cm} ; P<0.001$; $\mathrm{TE}=-1.55)$ (Figura 2). La altura de salto en partido 2 fue significativamente inferior que en partido $1(P<0.001$; TE $=0.61)$, partido $3(P=0.039$; TE $=0.56)$ y post-viaje $(P=0.029 ; \mathrm{TE}=0.53)$. No se observaron diferencias significativas en la altura de salto entre partido 1 , partido 3 y post-viaje. 
Figura 2. Cambio porcentual en la altura de salto respecto al pre-viaje. *, significativamente inferior al pre-viaje; \#, significativamente mayor al partido 2.

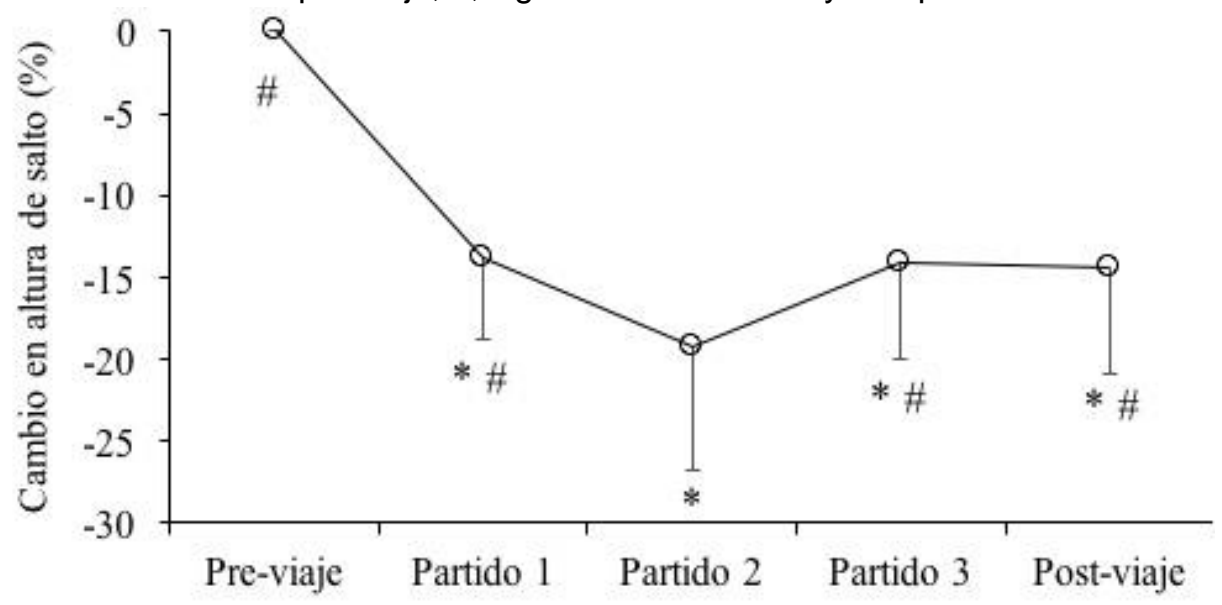

La diferencia en la altura de salto (post-viaje - pre-viaje) no mostró una asociación significativa ni con el total de minutos disputados $(r=-0.02 ; P=0.464)$ ni con la RPE media de los 3 partidos $(r=0.15 ; P=0.249)$ (Figura 3). El modelo de regresión lineal múltiple tampoco consiguió explicar una parte significativa de la varianza del cambio en la altura de salto $\left(r^{2}=0.03 ; P=0.751\right)$.

Figura 3. Asociación del cambio en la altura de salto (post-viaje - pre-viaje) con el total de minutos jugados (panel superior) y RPE media de los 3 partidos disputados (panel inferior).
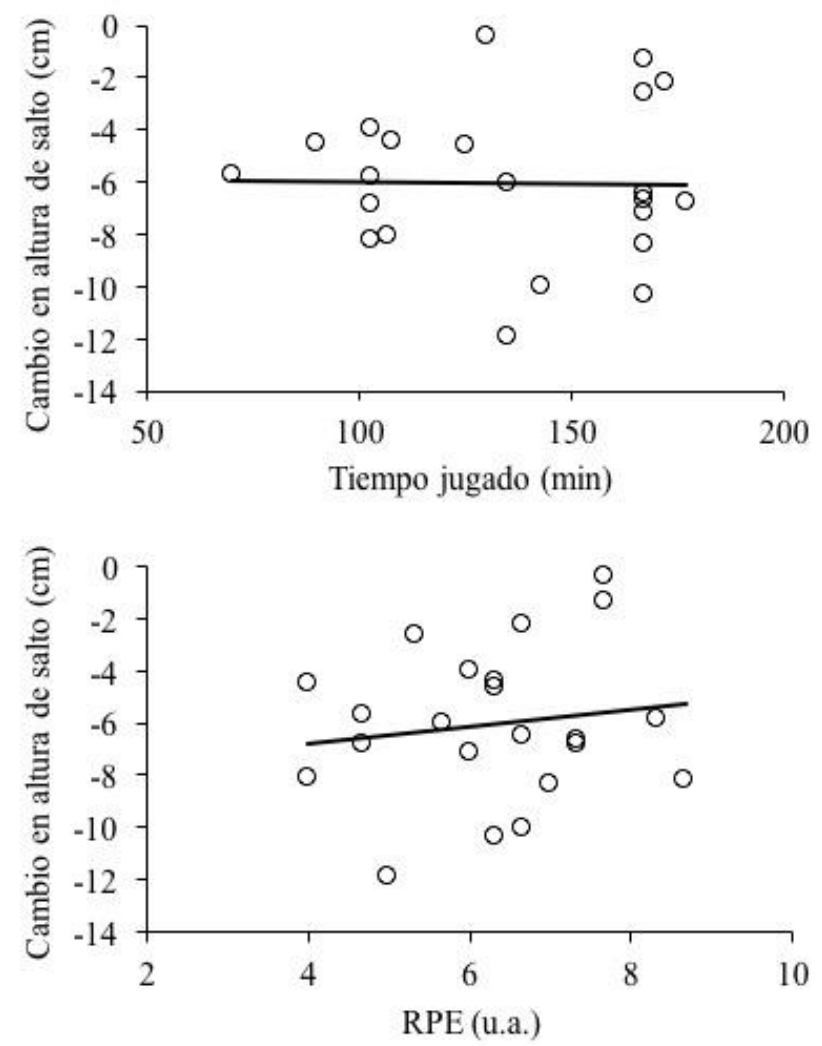


\section{DISCUSIÓN}

El presente estudio fue diseñado para comprobar si la disputa de varios partidos amistosos en días consecutivos y la realización de un viaje de larga duración en situaciones poco confortables podrían inducir cambios en la altura del salto vertical (CMJ). El principal hallazgo del presente estudio fue que la altura alcanzada en el CMJ se redujo de forma significativa desde la primera evaluación (pre-test) hasta la última evaluación (post-test), confirmándose así la primera hipótesis de nuestro estudio. No obstante, la segunda hipótesis no fue confirmada, ya que no se observó una correlación significativa entre el cambio en la altura del CMJ con respecto a los minutos totales disputados ni a la RPE media de los jugadores.

Las elevadas exigencias competitivas hacen necesario que los profesionales del deporte tengan un control de la carga de entrenamientos para prevenir lesiones, y con ello, mejorar el rendimiento deportivo. El CMJ es uno de los test de campo más utilizados para evaluar el estado neuromuscular y cuantificar la fatiga inducida por el entrenamiento en el deportista (Claudino et al., 2017; Gathercole, Sporer, Stellingwerff, \& Sleivert, 2015; Marqués-Jiménez et al., 2017; Watkins et al., 2017; Zurutuza, Castellano, Echeazarra, \& Casamichana, 2017). Diferentes variables cinéticas y cinemáticas pueden analizarse al evaluar el CMJ (McMahon, Suchomel, Lake, \& Comfort, 2018), siendo la altura de salto la más utilizada al poderse obtener de forma práctica y proporcionar información valiosa acerca del estado neuromuscular del deportista (Claudino et al., 2017). Por otro lado, para monitorizar y cuantificar la carga de entrenamiento también se utiliza la percepción subjetiva del esfuerzo (RPE), qué es un método válido, poco costoso, no invasivo y de fácil aplicación que nos permite conocer la carga interna de los deportistas (Campos-Vazquez et al., 2017; Foster et al., 2001; Impellizzeri, Rampinini, Coutts, Sassi, \& Marcora, 2004; Marqués-Jiménez et al., 2017). Por lo tanto, es interesante examinar si los resultados obtenidos entre estas dos variables podrían ir en paralelo, es decir, corroborar si un mayor decremento de la altura de salto del CMJ podría estar asociado con mayores valores de RPE o minutos disputados.

Nuestra primera hipótesis fue confirmada ya que la altura del salto vertical fue disminuyendo a medida que se iban disputando los partidos. En línea con los resultados obtenidos en nuestro estudio, Thomas et al. (2017) revelaron una disminución de la altura del salto vertical en futbolistas semi profesionales tras un partido de fútbol simulado. Del mismo modo, Watkins et al. (2017) mostraron resultados similares en deportistas que realizaron diferentes ejercicios que implicaban mayoritariamente a la musculatura inferior del cuerpo. No obstante, los resultados obtenidos en futbolistas de género femenino (Krustrup et al., 2010) y jóvenes varones (Thorlund et al., 2009), no concuerdan con los del presente estudio. Esto podría ser explicado por las diferencias en el desarrollo específico de la fatiga entre géneros 0 , incluso, por el nivel competitivo. Por lo tanto, los resultados del presente estudio confirman que la altura alcanzada en el CMJ 
puede ser un indicador válido para evaluar la fatiga inducida por varios partidos jugados en días consecutivos.

Otra posible explicación a la disminución del rendimiento en el salto vertical (CMJ) podría tener relación con el viaje de largo trayecto tanto en la ida como en la vuelta que realizaron los participantes en situaciones poco confortables. En base a esto, Chapman, Bullock, Ross, Rosemond, \& Martin (2012) analizaron el efecto de un viaje transmeridional en diferentes tests de salto durante varios días, observando una disminución significativa de la altura de salto en el CMJ después del viaje, coincidiendo con los resultados obtenidos en el presente estudio. No obstante, hay que tener cautela a la hora de interpretar estos resultados puesto que los medios de transportes utilizados en ambos estudios han sido diferentes (avión y autobús, respectivamente), así como, las situaciones de confortabilidad. Por tanto, existe la necesidad de que futuros estudios analicen los efectos de diferentes tipos de viaje (ej., autobús, tren, avión, etc.) sobre el estado neuromuscular del deportista.

Nuestra segunda hipótesis de estudio no fue confirmada puesto que no se observó una relación significativa entre los cambios de la altura del salto con los valores de RPE medios de los partidos disputados ni con la acumulación de minutos disputados. En concordancia con los resultados de este estudio, aunque utilizando el s-RPE respiratorio y muscular, Gil-Rey et al. (2015) no encontraron correlaciones significativas entre la carga de entrenamiento y el cambio en la altura del salto (CMJ) en equipos de jóvenes futbolistas de diferentes niveles competitivos (élite y no élite). A su vez, Arcos, Yanci, Mendiguchia, \& Gorostiaga (2014) han obtenido resultados similares en jugadores profesionales de fútbol al comparar el total de minutos acumulados (entrenamientos y partidos) y los cambios en la altura del salto durante 9 semanas. Serían necesarias más investigaciones al respecto para determinar si la fatiga neuromuscular inducida por varios partidos jugados en días consecutivos modifica la altura del salto vertical.

Varias limitaciones deberían mencionarse en este estudio: (1) la principal limitación fue que no se utilizó un grupo control para comparar los efectos del protocolo experimental propuesto en la investigación para observar diferencias inter-grupales; (2) no se utilizaron medidas objetivas para la cuantificación de la carga de entrenamiento. Futuras líneas de investigaciones podrían centrarse en la evaluación del rendimiento neuromusculares de jóvenes futbolistas de élite que disputan torneos en períodos pre-competitivo y competitivos en los cuales se disputan varios partidos en días consecutivos. Debido a la escasa bibliografía existente, sería interesante ver los efectos que provocan en el rendimiento neuromuscular y la recuperación de los deportistas un viaje en autobús, u otros medios de transporte más económicos, durante trayectos de larga duración. 


\section{CONCLUSIONES}

El presente estudio confirma que a través de la evaluación del salto de altura vertical (CMJ) es posible detectar la fatiga inducida por varios partidos de fútbol jugados en días consecutivos. Sin embargo, el decremento en la altura del CMJ no parece presentar una gran asociación ni con los valores de RPE ni con el total de minutos disputados. Los resultados obtenidos podrían ser de interés para los preparadores físicos al hacerles conscientes que un viaje en situaciones poco confortable de larga duración, encadenado con la disputa de varios partidos de fútbol en días consecutivos, puede comprometer el estado neuromuscular de los futbolistas, con el consiguiente riesgo de lesiones y disminución del rendimiento deportivo.

\section{REFERENCIAS BIBLIOGRÁFICAS}

Arcos, A. L., Yanci, J., Mendiguchia, J., \& Gorostiaga, E. M. (2014). Rating of Muscular and Respiratory Perceived Exertion in Professional Soccer Players. Journal of Strength and Conditioning Research, 28(11), 32803288. https://doi.org/10.1519/JSC.0000000000000540

Borg, G., Hassmén, P., \& Lagerström, M. (1987). Perceived exertion related to heart rate and blood lactate during arm and leg exercise. European Journal of Applied Physiology and Occupational Physiology, 56(6), 679-685. https://doi.org/10.1007/BF00424810

Brownstein, C. G., Dent, J. P., Parker, P., Hicks, K. M., Howatson, G., Goodall, S., \& Thomas, K. (2017). Etiology and Recovery of Neuromuscular Fatigue following Competitive Soccer Match-Play. Frontiers in Physiology, 8, 831. https://doi.org/10.3389/fphys.2017.00831

Campos-Vazquez, M. A., Toscano-Bendala, F. J., Mora-Ferrera, J. C., \& SuarezArrones, L. J. (2017). Relationship Between Internal Load Indicators and Changes on Intermittent Performance After the Preseason in Professional Soccer Players. Journal of Strength and Conditioning Research, 31(6), 1477-1485. https://doi.org/10.1519/JSC.0000000000001613

Chapman, D. W., Bullock, N., Ross, A., Rosemond, D., \& Martin, D. T. (2012). Detrimental effects of West to East transmeridian flight on jump performance. European Journal of Applied Physiology, 112(5), 1663-1669. https://doi.org/10.1007/s00421-011-2134-6

Claudino, J. G., Cronin, J., Mezêncio, B., McMaster, D. T., McGuigan, M., Tricoli, V., ... Serrão, J. C. (2017). The countermovement jump to monitor neuromuscular status: A meta-analysis. Journal of Science and Medicine in Sport, 20(4), 397-402. https://doi.org/10.1016/j.jsams.2016.08.011

de Hoyo, M., Cohen, D. D., Sañudo, B., Carrasco, L., Álvarez-Mesa, A., del Ojo, J. J., ... Otero-Esquina, C. (2016). Influence of football match time-motion parameters on recovery time course of muscle damage and jump ability. Journal of Sports Sciences, 34(14), 1363-1370. https://doi.org/10.1080/02640414.2016.1150603

Faude, O., Koch, T., \& Meyer, T. (2012). Straight sprinting is the most frequent action in goal situations in professional football. Journal of Sports Sciences, 
30(7), 625-31. https://doi.org/10.1080/02640414.2012.665940

Foster, C., Florhaug, J. a, Franklin, J., Gottschall, L., Hrovatin, L. a, Parker, S., ... Dodge, C. (2001). A new approach to monitoring exercise training. Journal of Strength and Conditioning Research, 15(1), 109-115. https://doi.org/10.1519/1533-4287(2001)015<0109:ANATME>2.0.CO;2

Fowler, P., Duffield, R., \& Vaile, J. (2015). Effects of simulated domestic and international air travel on sleep, performance, and recovery for team sports. Scandinavian Journal of Medicine \& Science in Sports, 25(3), 441-451. https://doi.org/10.1111/sms. 12227

Gathercole, R. J., Sporer, B. C., Stellingwerff, T., \& Sleivert, G. G. (2015). Comparison of the Capacity of Different Jump and Sprint Field Tests to Detect Neuromuscular Fatigue. Journal of Strength and Conditioning Research, 29(9), 2522-2531. https://doi.org/10.1519/JSC.0000000000000912

Gil-Rey, E., Lezaun, A., \& Los Arcos, A. (2015). Quantification of the perceived training load and its relationship with changes in physical fitness performance in junior soccer players. Journal of Sports Sciences, 33(20), 2125-2132. https://doi.org/10.1080/02640414.2015.1069385

Hopkins, W. G., Marshall, S. W., Batterham, A. M., \& Hanin, J. (2009). Progressive statistics for studies in sports medicine and exercise science. Medicine and Science in Sports and Exercise, 41(1), 3-13. https://doi.org/10.1249/MSS.0b013e31818cb278

Impellizzeri, F. M., Rampinini, E., Coutts, A. J., Sassi, A., \& Marcora, S. M. (2004). Use of RPE-based training load in soccer. Medicine and Science in Sports and Exercise, 36(6), 1042-7. Retrieved from http://www.ncbi.nlm.nih.gov/pubmed/15179175

Ispirlidis, I., Fatouros, I. G., Jamurtas, A. Z., Nikolaidis, M. G., Michailidis, I., Douroudos, I., ... Taxildaris, K. (2008). Time-course of Changes in Inflammatory and Performance Responses Following a Soccer Game. Clinical Journal of Sport Medicine, 18(5), 423-431. https://doi.org/10.1097/JSM.0b013e3181818e0b

Jiménez-Reyes, P., Samozino, P., Pareja-Blanco, F., Conceição, F., CuadradoPeñafiel, V., González-Badillo, J. J., \& Morin, J. B. (2017). Validity of a simple method for measuring force-velocity-power profile in countermovement jump. International Journal of Sports Physiology and Performance, 12(1), 36-43. https://doi.org/10.1123/IJSPP.2015-0484

Krustrup, P., Zebis, M., Jensen, J. M., \& Mohr, M. (2010). Game-Induced Fatigue Patterns in Elite Female Soccer. Journal of Strength and Conditioning Research, 24(2), 437-441. https://doi.org/10.1519/JSC.0b013e3181c09b79

Malone, J. J., Di Michele, R., Morgans, R., Burgess, D., Morton, J. P., \& Drust, B. (2015). Seasonal Training-Load Quantification in Elite English Premier League Soccer Players. International Journal of Sports Physiology and Performance, 10(4), 489-497. https://doi.org/10.1123/ijspp.2014-0352

Manzi, V., Bovenzi, A., Franco Impellizzeri, M., Carminati, I., \& Castagna, C. (2013). Individual Training-Load and Aerobic-Fitness Variables in Premiership Soccer Players During the Precompetitive Season. Journal of Strength and Conditioning Research, 27(3), 631-636. 
https://doi.org/10.1519/JSC.0b013e31825dbd81

Marqués-Jiménez, D., Calleja-González, J., Arratibel, I., Delextrat, A., \& Terrados, N. (2017). Fatigue and Recovery in Soccer: Evidence and Challenges. The Open Sports Sciences Journal, 10(Suppl 1: M5), 52-70. https://doi.org/10.2174/1875399X01710010052

McMahon, J. J., Suchomel, T. J., Lake, J. P., \& Comfort, P. (2018). Understanding the Key Phases of the Countermovement Jump Force-Time Curve. Strength and Conditioning Journal, 1. https://doi.org/10.1519/SSC.0000000000000375

Roe, G., Darrall-Jones, J., Till, K., Phibbs, P., Read, D., Weakley, J., \& Jones, B. (2017). To Jump or Cycle? Monitoring Neuromuscular Function in Rugby Union Players. International Journal of Sports Physiology and Performance, 12(5), 690-696. https://doi.org/10.1123/ijspp.2016-0273

Scott, B. R., Lockie, R. G., Knight, T. J., Clark, A. C., \& Janse de Jonge, X. A. K. (2013). A comparison of methods to quantify the in-season training load of professional soccer players. International Journal of Sports Physiology and Performance, 8(2), 195-202. Retrieved from http://www.ncbi.nlm.nih.gov/pubmed/23428492

Silva, J. R., Rumpf, M. C., Hertzog, M., Castagna, C., Farooq, A., Girard, O., \& Hader, K. (2017). Acute and Residual Soccer Match-Related Fatigue: A Systematic Review and Meta-analysis. Sports Medicine (Auckland, N.Z.). https://doi.org/10.1007/s40279-017-0798-8

Thomas, K., Dent, J., Howatson, G., \& Goodall, S. (2017). Etiology and Recovery of Neuromuscular Fatigue after Simulated Soccer Match Play. Medicine and Science in Sports and Exercise, 49(5), 955-964. https://doi.org/10.1249/MSS.0000000000001196

Thorlund, J. B., Aagaard, P., \& Madsen, K. (2009). Rapid muscle force capacity changes after soccer match play. International Journal of Sports Medicine, 30(4), 273-8. https://doi.org/10.1055/s-0028-1104587

Vanrenterghem, J., Nedergaard, N. J., Robinson, M. A., \& Drust, B. (2017). Training Load Monitoring in Team Sports: A Novel Framework Separating Physiological and Biomechanical Load-Adaptation Pathways. Sports Medicine, 47(11), 2135-2142. https://doi.org/10.1007/s40279-017-0714-2

Watkins, C. M., Barillas, S. R., Wong, M. A., Archer, D. C., Dobbs, I. J., Lockie, R. G., ... Brown, L. E. (2017). Determination of Vertical Jump as a Measure of Neuromuscular Readiness and Fatigue. Journal of Strength and Conditioning Research, 31(12), 3305-3310. https://doi.org/10.1519/JSC.0000000000002231

Zurutuza, U., Castellano, J., Echeazarra, I., \& Casamichana, D. (2017). Absolute and Relative Training Load and Its Relation to Fatigue in Football. Frontiers in Psychology, 8, 878. https://doi.org/10.3389/fpsyg.2017.00878

Referencias totales / Total references: 29 (100\%)

Referencias propias de la revista / Journal's own references: 0 (0\%)

Rev.int.med.cienc.act.fís.deporte - vol. X - número X - ISSN: 1577-0354 